УДК 616.24-002.51

DOI: 10.15587/2313-8416.2015.38158

\title{
ИЗМЕНЕНИЯ В ЛЕГОЧНОЙ ТКАНИ, СВЯЗАННЫЕ С АХАЛАЗИЕЙ ПИЩЕВОДА. КЛИНИЧЕСКИЙ СЛУЧАЙ
}

\author{
(ㄷ) А. А. Ляшенко
}

Ахалазия пищевода является идиопатическим заболеванием легких, сопровождается нарастающей дисфагией и дилатаџией пищевода. Наиболее частым осложнением со стороны дыхательной системы является аспираџионная пневмония, но иногда может присоединяться и туберкулез легких. Лечение туберкулеза легких у таких пациентов может быть неэффективно из-за нарушения прохождения пищи, и, соответственно лекарственных вещзеств. В связи с этим больным туберкулезом легких на фоне ахалазии пищевода рекомендовано инъекционное введение лекарственных веществ

Ключевые слова: ахалазия пищевода, туберкулез легких, клинический случай, лечение туберкулеза, аспирациинная пневмония

Esophageal achalasia is a disease of unknown etiology, accompanied by the motility disorder, which leads to the esophageal food impaction. According to statistics, the incidence of esophageal achalasia is only 1 case per 100000 population. Despite the fact that the disease is quite rare, physician-pulmonologist still meets such patients in the practice. At night, aggressive gastric contents in patients with esophageal achalasia can enter the respiratory tract, causing mostly aspiration pneumonia, less often - pulmonary tuberculosis. Features of treatment of pulmonary tuberculosis with esophageal achalasia are under-investigated, the problem is urgent.

Objective: to analyze the characteristics of pulmonary tuberculosis in patients with esophageal achalasia, to optimize the treatment regimens for such patients.

Methods: from 2008 to 2012, we observed 4 cases of esophageal achalasia patients with pulmonary disease as a complication. All patients had recurrent pneumonia. One patient with a history of frequent pneumonia had pulmonary tuberculosis.

Result: treatment of pulmonary tuberculosis with concomitant esophageal achalasia is difficult in some cases because of the food impaction in the gastrointestinal tract. As a result, a standard regimen of tuberculosis treatment by oral drugs may be ineffective, and parenteral or intramuscular administration of antituberculosis drugs is recommended in this case.

Conclusion: injection of anti-TB drugs is mainly recommended for pulmonary tuberculosis patients with concomitant esophageal achalasia

Keywords: esophageal achalasia, pulmonary tuberculosis, clinical case, treatment of tuberculosis, aspiration pneumonia

\section{1. Введение}

Ахалазия пищевода (кардиоспазм, мегаэзофагус) - заболевание, характеризующееся нарушением перистальтики пищевода в результате снижения тонуса мышечного слоя и отсутствия полного расслабления нижнего пищеводного сфинктера (НПС) при глотании, проявляющееся нарушением прохождения пищи по пищеводу [1].

Распространенность ахалазии во всех странах приблизительно одинакова и составляет 0,5-1 на 100000 населения [2, 3]. Пик заболеваемости приходится на возраст 20-40 лет. «Золотым» стандартом диагностики ахалазии пищевода является манометрия, в то время как наличие симптомов, ендоскопическое и исследование с бариевой взвесью признано недостаточно эффективными [4]. На основе высокоразрешающей манометрии выделяют несколько типов ахалазии и имеется возможность дифференцировать это заболевание с другими заболеваниями пищевода и нарушениями пищеварения. Для пациентов старше 45 лет рекомендуется дополнительно проводить эндоспоки- ческую ультразвуковую диагностику и компьютерную томографию для исключения псевдоахалазии.

\section{2. Литературный обзор}

Наиболее частым проявлением ахалазии является дисфагия, несколько реже встречается регургитация (60\%), боль в грудной клетке (40 \%), которая обычно возникает во время еды и изжога (у 40 \%), боль в грудной клетке и потеря массы тела $[1,5,6]$. Регургитация чаще возникает в положении лежа на спине, что может приводить к аспирации непереваренной пищи. При рентгеноконтрастном исследовании обнаруживается деформация и расширение пищевода в виде «клюва птицы». Наиболее информативным методом выявления ахалазии пищевода является компьютерная томография [7]. Лечение больных ахалазией включают фармакологическую терапию, эндоскопическую инъекцию токсина ботулина, пневматическую дилатацию и миотомию (сначала выполнялась лапароскопически, на более поздних этапах - эндоскопическим путем) [8]. Фармакологическая терапия показала себя неэф- 
фективной при этом заболевании и обладала высокой тоскичностью. Инъекции токсином ботулина безопасны, но эффект оказался непродолжительным, эффект уменьшается со временем и почти исчезает уже через несколько месяцев после лечения. Самыми эффективными методами лечения на сегодня являются пневматическое расширение и хирургическая миотомия, эффективность которых доказана продолжительным наблюдением за пациентами более двух лет [9].

Нарушение перистальтики и отсутствие расслабления НПС являются причиной застоя пищевых масс и заброса желудочного сока в пищевод. Наиболее частым осложнением является эзофагит, который обнаруживается у большинства больных с ахалазией. Около 30 \% пациентов с ахалазией предъявляют жалобы на ночные эпизоды кашля, у таких больных часто возникает аспирационная пневомния [7, 10]. Ахалазия пищевода также рассматривается как фактор риска развития туберкулеза легких, аспирационной пневмонии, бронхиальной астмы, бронхоэктазов и др. $[1,11,12]$.

В доступной литературе чаще всего встречается не найдено описания рекомендаций по лечению пациентов туберкулезом легких в сочетании с ахалазией пищевода.

\section{3. Материалы и методы}

В период с 2008 по 2012 года под наблюдением было четыре больных с ахалазией пищевода (2-х женщин и 2-х мужчин). Все больные были старше 30 лет. В анамнезе у всех больных отмечались повторные аспирационные пневмонии, по поводу которых проводилось лечение антибиотиками широкого спектра действия, как правило, амбулаторно. У одной больной выраженная ахалазия пищевода осложнилась частыми повторными аспирационными пневмониями и рецидивирующим туберкулезом.

\section{4. Результаты исследования}

Приводим клинический случай.

Больная Б., 45 лет. В возрасте 30 лет после стресса сильно похудела (на 12 кг в течение нескольких месяцев). При рентгенологическом исследовании была обнаружена ахалазия пищевода, в связи с чем больной проведено однократное бужирование. При рентгеноскопии желудочно-кишечного тракта с контрастированием установлена ахалазия II-III степени. Неоднократно лечилась амбулаторно по поводу пневмоний.

При очередном профилактическом осмотре выявлены изменения в легких. После дообследования установлен диагноз: впервые диагностированный туберкулез легких (17.03.2005) правого легкого (инфильтративный), деструкции легочной ткани не обнаружено, микобактерии в мокроте найдены микроскопически и методом посева на твердые и жидкие питательные среды, микобактерии туберкулеза были чувствительны ко всем противотуберкулезным препаратам, лечение начали согласно действующим законам и приказам Украины. Пролечена по стандартной схеме в течение 6 месяцев (категория 1) с положительным эффектом - отмечалось прекращение бактериовыделения и положительная рентгенологическая динамика в виде частичного рассасывания инфильтративных изменений в легких. Была переведена в категорию больных с остаточными изменениями излеченного туберкулеза (Кат. 5, группа 5.1.).

Через три года при очередном профилактическом осмотре выявлена отрицательная динамика в виде нарастания инфильтративных изменений в обоих легких. Для дообследования и уточнения диагноза направлена в стационар. Жаловалась на кашель со скудной слизисто-гнойной мокротой, чувство задержки пищи в пищеводе, отрыжку. Аускультативно в межлопаточной области слева немногочисленные среднепузырчатые влажные и сухие хрипы.

В мокроте методом посева обнаружены МБТ.

В клиническом анализе крови отмечалось увеличение СОЭ до 30 мм/час, остальные показатели крови - в пределах нормы.

Общий анализ мочи - без особенностей.

На обзорной рентгенограмме выявлены инфильтративные тени в обоих легких преимущественно в средних и нижних отделах (рис. 1).

Клинический диагноз: рецидив туберкулеза (08.10.2009) легких (инфильтративный), Дестр-, МБТ+, М-, К+, Резист 0, Гист 0, Кат 2, Ког 4 (2009). Начато лечение по категории 2 пятью противотуберкулезными препаратами. Несмотря на проводимую терапию состояние пациентки ухудшилось, на 3-м месяце лечения у больной усилился кашель, повысилась температура тела до $38,5^{\circ} \mathrm{C}$. В легких, преимущественно над нижней долей левого легкого, выслушивались множественные разнокалиберные влажные хрипы. На компьютерной томограмме легких от 25.12.2009: определяется выраженное расширение пищевода на исследуемом уровне на всем протяжении до кардиоэзофагального перехода (максимальный диаметр до 60 мм) с большим количеством содержимого. Слева в $\mathrm{S}_{6}$ и $\mathrm{S}_{10}$ визуализируются множественные участки мягкотканой плотности сливного характера размерами до 37 мм, на фоне которых прослеживаются воздушные бронхи. В верхних долях с обеих сторон и в средней доле справа аналогичной структуры, меньших размеров образования размерами до 17 мм (рис. 2).

Диагностированы прогрессирование туберкулеза и аспирационная пневмония.

В связи с наличием ахалазии пищевода и сохраняющимся неудовлетворительным состоянием пациентки, принято решение заменить режим противотуберкулезной терапии на индивидуализированное лечение (все протипотуберкулезные препараты назначены парентерально). Проведен также курс лечения далацином Ц с эффектом.

После окончания основного курса АМБТ достигнуто прекращение бактериовыделения, при контрольном рентгенологическом обследовании отмечена положительная динамика в виде значительного рассасывания инфильтративных теней в обоих легких (рис. 3). 


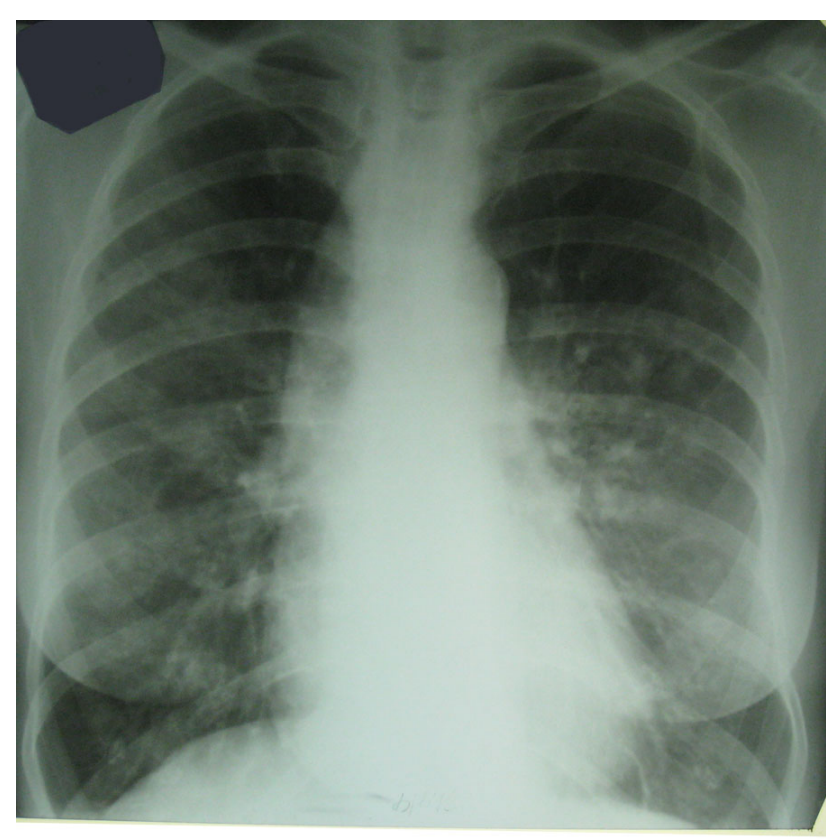

Рис. 1. Больная Б. Обзорная рентгенограмма органов грудной клетки от 24.09.2009
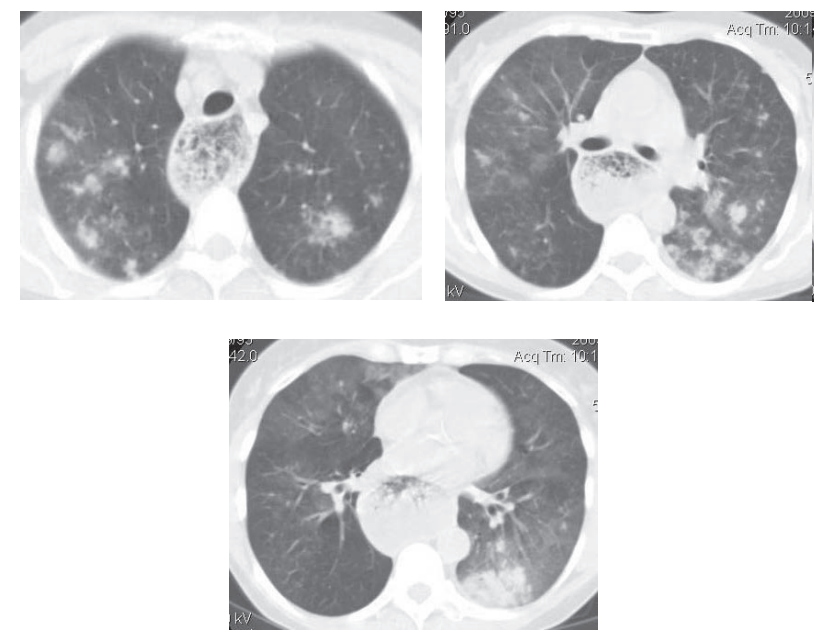

Рис. 2. Больная Б. Компьютерная томограмма от 25.12.2009

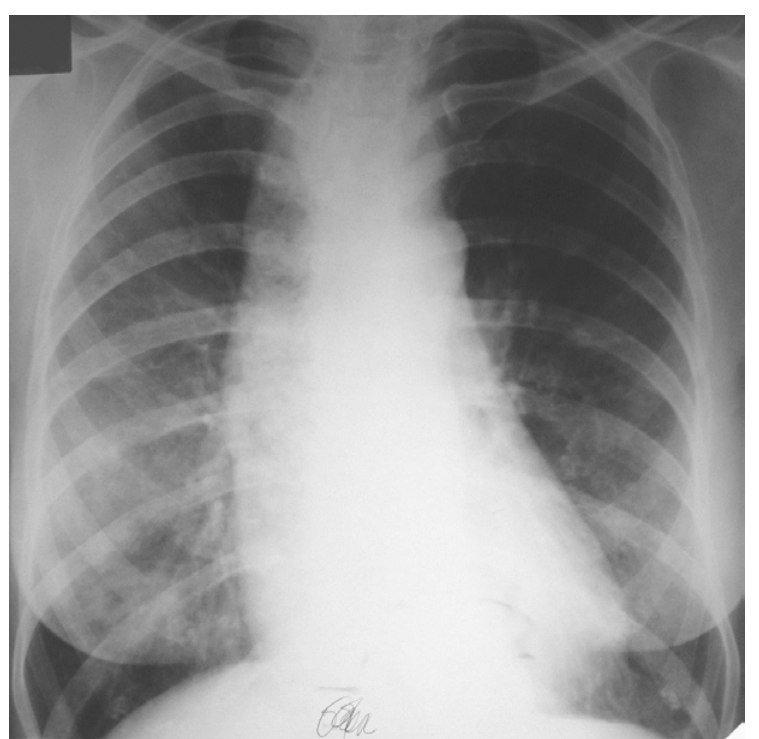

Рис. 3. Больная Б. Обзорная рентгенограмма органов грудной клетки от 29.04.2010
В конце интенсивной фазы, больная переведена в амбулаторное отделение для дальнейшего наблюдения и лечения у фтизиатра и гастроэнтеролога.

\section{5. Выводы}

Наиболее частым осложнением ахалазии пищевода является аспирационная пневмония, однако, не стоит забывать высокую вероятность развития туберкулеза легких. При лечении туберкулеза легких в таких случаях рекомендуется использовать парентеральный путь введения препаратов.

\section{Литература}

1. Василенко, В. Х. Болезни желудка, кишечника и брюшины [Текст] / под ред. В. Х. Василенко; пер. с болг. София: Медицина и физкультура, 1964. - 784 с.

2. Mayberry, J. F. Achalasia in the city of Cardiff from 1926 to 1977 [Text] / J. F. Mayberry, J. Rhodes // Digestion. 1980. - Vol. 20, Issue 4. - P. 248-252. doi: 10.1159/000198446

3. Mayberry, J. F. Studies of incidence and prevalence of achalasia in the Nottingham area [Text] / J. F. Mayberry, M. Atkinson // Q J Med. - 1985. - Vol. 220, Issue 56. - P. 451-456.

4. von Rahden, B. H. Diagnostics and therapy of achalasia [Text] / B. H. von Rahden, J. Filser, F. Seyfried et al. // Chirurg. - 2014. - Vol. 85, Issue 12. - P. 1055-1063. doi: 10.1007/ s00104-014-2803-1

5. Василенко, В. Х. Ахалазия кардии [Текст] / В. Х. Василенко, Т. А. Суворова, А. Л. Гребенев. - М: Медицина, 1976. $-280 \mathrm{c}$.

6. Barajas-Fregoso, E. M. Experience of the surgical management of the esophageal achalasia in a tertiary care hospital [Text] / E. M. Barajas-Fregoso, T. Romero-Hernández, P. R. Sánchez-Fernández et al. // Rev Med Inst Mex Seguro Soc. - 2015. - Vol. 53, Issue 11. - P. 84-91.

7. Шептулин, А. А. Новое в диагностике и лечение гастроэзофагальной болезни и ахалазии кардии [Текст] / А. А. Шептулин, А. С. Трухманов // Клин. мед. - 1998. № 5. - C. 15-19.

8. Vela, M. F. Management strategies for achalasia [Text] / M. F. Vela // Neurogastroenterology \& Motility. - 2014. Vol. 29, Issue 9. - P. 1215-1221. doi: 10.1111/nmo.12416

9. Yasawy, M. I. Diagnosis and treatment of achalasia patients: a ten-year review of the clinical features [Text] / M. I. Yasawy // Hepatogastroenterology. - 2014. - Vol. 61, Issue 134. - P. 1611-1616.

10. Ивашкина, В. Т. Гастроэнтерология. Национальное руководство: краткое издание [Текст] / под ред. В. Т. Ивашкина, Т. Л. Лапиной. - М.: ГЭОТАР-Медиа, 2011. - 480 с.

11. Layton, J. Acute respiratory failure secondary to esophageal dilation from undiagnosed achalasia [Text] / J. Layton, P. W. Ward, D. W. Miller, R. M. Roan // A A Case Rep. 2014. - Vol. 3, Issue 5. - P. 65-67.

12. Hirata, M. A case of recurrent aspiration pneumonia by achalasia [Text] / M. Hirata, K. Kohno, S. Murakami et al. // Nihon Kokyuki Gakkai Zasshi. - 2002. - Vol. 40, Issue 2. P. 149-153.

\section{References}

1. Vasilenko, V. H. (Ed.) (1964). Bolezni zheludka, kishechnika i brjushiny. Sofija: Medicina i fizkul'tura, 784.

2. Mayberry, J. F., Rhodes, J. (1980). Achalasia in the City of Cardiff from 1926 to 1977 . Digestion, 20 (4), 248-252. doi: $10.1159 / 000198446$

3. Mayberry, J. F., Atkinson, M. (1985). Studies of incidence and prevalence of achalasia in the Nottingham area. Q J Med., 220 (56), 451-456. 
4. von Rahden, B. H., Filser, J., Seyfried, F. et al. (2014). Diagnostics and therapy of achalasia. Chirurg, 85 (12), 1055-1063. doi: 10.1007/s00104-014-2803-1

5. Vasilenko, V. X., Suvorova, T. A., Grebenev, A. L. (1976). Ahalazija kardii. Moscow: Medicina, 280.

6. Barajas-Fregoso, E. M., Romero-Hernández, T., Sánchez-Fernández, P. R. et al. (2015). Experience of the surgical management of the esophageal achalasia in a tertiary care hospital. Rev Med Inst Mex Seguro Soc., 53 (11), 84-91.

7. Sheptulin, A. A., Truhmanov, A. S. (1998). Novoe v diagnostike i lechenie gastrojezofagal'noj bolezni i ahalazii kardii. Klin. med., 5, 15-19.

8. Vela, M. F. (2014). Management strategies for achalasia. Neurogastroenterology \& Motility, 26 (9), 12151221. doi: $10.1111 / \mathrm{nmo} .12416$
9. Yasawy, M. I. (2014). Diagnosis and treatment of achalasia patients: a ten-year review of the clinical features. Hepatogastroenterology, 61 (134), 1611-1616.

10. Ivashkina, V. T., Lapinoj, T. L. (Eds.) (2011). Gastrojenterologija. Nacional'noe rukovodstvo: kratkoe izdanie. Moscow: GJeOTAR-Media, 480.

11. Layton, J., Ward, P. W., Miller, D. W., Roan, R. M. (2014). Acute respiratory failure secondary to esophageal dilation from undiagnosed achalasia. A A Case Rep., 3 (5), 65-67.

12. Hirata, M., Kohno, K., Murakami, S. et al. (2002). A case of recurrent aspiration pneumonia by achalasia. Nihon Kokyuki Gakkai Zasshi, 40 (2), 149-153.

Рекомендовано до публікачії д-р мед. наук, професор Крутько В. С. Дата надходження рукопису 16.01.2015

Ляшенко Александр Алексеевич, кандидат медицинских наук, доцент, кафедра фтизиатрии и пульмонологии, Харьковская медицинская академия последипломного образования, ул. Корчагинцев 58, г. Харьков, Украина, 61176

E-mail: thepulmonolog@gmail.com 